\title{
Diverging dc conductivity due to a flat band in a disordered system of pseudospin-1 Dirac-Weyl fermions
}

\author{
Máté Vigh, ${ }^{1}$ László Oroszlány, ${ }^{2}$ Szabolcs Vajna, ${ }^{3}$ Pablo San-Jose, ${ }^{4}$ Gyula Dávid, ${ }^{1}$ József Cserti, ${ }^{1}$ and Balázs Dóra ${ }^{3, *}$ \\ ${ }^{1}$ Department of Physics of Complex Systems, Eötvös University, H-1117 Budapest, Hungary \\ ${ }^{2}$ Department of Theoretical Physics, Budapest University of Technology and Economics, Budapest, Hungary \\ ${ }^{3}$ Department of Physics and BME-MTA Exotic Quantum Phases Research Group, Budapest University of Technology \\ and Economics, Budapest, Hungary \\ ${ }^{4}$ Instituto de Ciencia de Materiales de Madrid (ICMM-CSIC), Cantoblanco, 28049 Madrid, Spain
}

(Received 4 June 2013; published 30 October 2013)

\begin{abstract}
Several lattices, such as the dice or the Lieb lattice, possess Dirac cones and a flat band crossing the Dirac point, whose effective model is the pseudospin-1 Dirac-Weyl equation. We investigate the fate of the flat band in the presence of disorder by focusing on the density of states (DOS) and dc conductivity. While the central hub site does not reveal the presence of the flat band, the sublattice resolved DOS on the noncentral sites exhibits a narrow peak with height $\sim 1 / \sqrt{g}$ with $g$ the dimensionless disorder variance. Although the group velocity is zero on the flat band, the de conductivity diverges as $\ln (1 / g)$ with decreasing disorder due to interband transitions around the band touching point between the propagating and the flat band. Generalizations to higher pseudospin are given.
\end{abstract}

DOI: 10.1103/PhysRevB.88.161413

\section{INTRODUCTION}

Flat bands are at the heart of several peculiar phenomena in condensed matter, especially in the presence of strong correlations. Prominent examples include (nearly) flat band ferromagnetism, integer and fractional quantum Hall effect arising from Landau levels in finite magnetic field, and, recently, fractional quantum Hall effect at zero magnetic field. ${ }^{1-3}$ Engineering flat bands with topologically nontrivial character has become a major challenge recently in connection with topological insulators. ${ }^{4,5}$

In addition to flat bands as surface modes, these also appear as bulk bands in systems with specific two-dimensional lattice structures, such as the dice or $T_{3}$ lattices, the Lieb lattice, etc. The common feature in the spectrum of these lattices is a graphene-like Dirac cone, intersected by a completely dispersionless flat band at the Dirac point (see Fig. 1). These can be regarded as the pseudospin-1 generalization of the Dirac equation, ${ }^{6-10}$ and arise in the family of higher pseudospin generalizations of the Dirac equation, proposed in Refs. 11-13.

While many of their properties are well understood, including topology, not much is known about their transport properties, ${ }^{10}$ which promise many excitement in light of the fascinating transport properties of their pseudospin- $1 / 2$ counterpart in graphene. There, the universal value of the minimal conductivity at half-filling ${ }^{14}$ attracted significant attention over the years, whereby the decreasing number of charge carriers as the charge neutrality point is approached exactly compensates their increasingly long lifetime.

Charge transport in the pseudospin-1 family of Dirac-Weyl fermions seems to be nontrivial as well. Due to the flat band, the density of states (DOS) exhibits a sharp peak at the neutrality point, though the group velocity on the flat band is identically zero. Consequently, at least two scenarios seem plausible for the behavior of the dc conductivity: it can remain insensitive to the flat band or it can be influenced by the large number of available states on the flat band. Intriguingly, none of these simple pictures are completely correct: while
PACS number(s): 05.60.Gg, 05.30.Fk, 71.10.Fd, 72.80.Vp the dc conductivity solely from the flat band vanishes due its zero velocity, interband transition between the flat and adjacent propagating bands are possible at the band touching degeneracy point, and this transition causes the divergence of the dc conductivity in the pure system precisely due to the zero flat band velocity. In the presence of disorder, this divergence is cut off logarithmically by the disorder strength, as we show by a careful numerical and analytical investigation of the problem.

Experimentally, the dice lattice can be realized from a trilayer structure of the face-centred cubic lattice, grown in the [111] direction. ${ }^{13}$ In particular, $\mathrm{SrTiO}_{3} / \mathrm{SrIrO}_{3} / \mathrm{SrTiO}_{3}$ trilayer heterostructures ${ }^{15}$ were found to realize the dice lattice structure, though further ab initio studies are desirable to decide how faithfully it can be described by Eq. (1). Additionally, the dice or Lieb lattices can be created via optical means in the cold atomic setting, and disorder can be introduced in a controlled manner using speckle potentials ${ }^{16,17}$ or by using holographic mask, enabling arbitrary geometries, to generate the desired lattice structure with disorder. ${ }^{18}$ While the local DOS has long been measurable by, e.g., time of flight imaging or $\mathrm{rf}$ spectroscopy, ${ }^{16}$ the dc conductivity is also accessible in this setting as well. ${ }^{19}$ Although cold atoms are neutral, the equivalent of an elecric potential can easily be created by tilting the trapping potential or by accelerating the lattice. In addition, the current between two macroscopic, density imbalanced reservoirs, connected by a narrow channel was investigated recently. ${ }^{19}$ This opens the way towards quantum transport simulation with quantum gases.

\section{DENSITY OF STATES}

The dice lattice, shown in Fig. 1, consists of a sixfold coordinated hub site $(H)$ and two threefold coordinated rim sites $(A$ and $B$ ) within its unit cell, with uniform hopping integrals $t$. Its Brillouin zone is hexagonal, and contains low-energy excitations close to zero energy at the two nonequivalent corners of the Brillouin zone, ${ }^{6}$ similarly to graphene ${ }^{20}$ These 

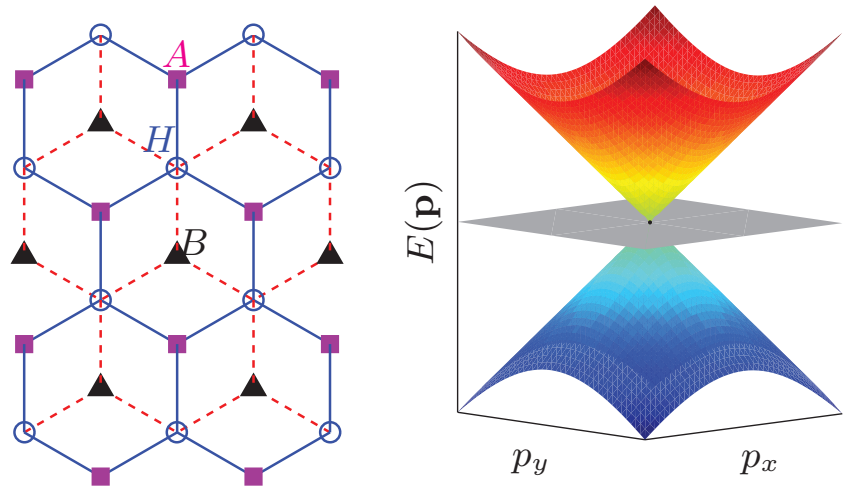

FIG. 1. (Color online) A small segment of the dice or $T_{3}$ lattice, consisting of two, one sublattice sharing (circles, hub site) honeycomb lattices, is shown (left), where the lines denote uniform hoppings $t$, together with its low-energy spectrum around the corners of the hexagonal Brillouin zone, featuring a flat band at the touching point of the Dirac cones (right).

are described by the pseudospin-1 Dirac-Weyl equation as ${ }^{13}$

$$
H_{0}=v_{F} \mathbf{S} \cdot \mathbf{p},
$$

where $v_{F}$ is the Fermi velocity, $\mathbf{p}=\left(p_{x}, p_{y}\right)$ and

$$
S_{x}=\frac{1}{\sqrt{2}}\left(\begin{array}{ccc}
0 & 1 & 0 \\
1 & 0 & 1 \\
0 & 1 & 0
\end{array}\right), \quad S_{y}=\frac{1}{\sqrt{2}}\left(\begin{array}{ccc}
0 & -i & 0 \\
i & 0 & -i \\
0 & i & 0
\end{array}\right)
$$

The resulting band-structure consists of three bands as $E_{ \pm}(\mathbf{p})= \pm v_{F}|p|$ and $E_{0}(\mathbf{p})=0$. The density of states (DOS) reads as

$$
\rho(\omega)=\frac{A_{c}}{2 \pi} \frac{|\omega|}{v_{F}^{2}}+\delta(\omega)=\frac{2|\omega|}{D^{2}}+\delta(\omega)
$$

for $|\omega|<D$ per spin, valley and unit cell, $A_{c}=4 \pi / k_{c}^{2}$ being the unit cell area. Here, $k_{c}$ is the momentum space cutoff and $D=v_{F} k_{c}$ is the half-bandwidth. The DOS satisfies $\int_{-D}^{D} d \omega \rho(\omega)=3$ and remains linear in energy close to halffilling, similar to graphene, but exhibits a sharp peak due to the flat band ${ }^{6}$ at zero energy.

The effect of weak disorder is modeled by adding a shortrange Gaussian potential as

$$
U(\mathbf{r})=\left(\begin{array}{ccc}
U_{A}(\mathbf{r}) & 0 & 0 \\
0 & U_{H}(\mathbf{r}) & 0 \\
0 & 0 & U_{B}(\mathbf{r})
\end{array}\right),
$$

where $\overline{U_{A}(\mathbf{r}) U_{A}\left(\mathbf{r}^{\prime}\right)}=U^{2} \delta\left(\mathbf{r}-\mathbf{r}^{\prime}\right)$ (and similarly for sublattice $H$ and $B$ ) with no intersublattice disorder correlation. The overline represents disorder averaging. To determine the structure of the self-energy, we study the effect of disorder within the self-consistent Born approximation (SCBA) as

$$
\Sigma\left(i \omega_{n}\right)=\frac{U^{2}}{N} \sum_{\mathbf{p}} G\left(\mathbf{p}, i \omega_{n}\right),
$$

where $N$ is the number of unit cells,

$$
G\left(\mathbf{p}, i \omega_{n}\right)=\left[i \omega_{n}-H_{0}-\Sigma\left(i \omega_{n}\right)\right]^{-1},
$$

and $\omega_{n}$ is the fermionic Matsubara frequency. The off-diagonal elements of the Green's function vanish after momentum integration, and the self-energy reads as

$$
\Sigma\left(i \omega_{n}\right)=\left(\begin{array}{ccc}
\Sigma_{A}\left(i \omega_{n}\right) & 0 & 0 \\
0 & \Sigma_{H}\left(i \omega_{n}\right) & 0 \\
0 & 0 & \Sigma_{A}\left(i \omega_{n}\right)
\end{array}\right) .
$$

The self-energy highlights the distinct structure of sublattice $H$, i.e., hopping from $A$ to $B$ is only possible through $H$. The self-consistency equations are expressed as

$$
\begin{aligned}
& \Sigma_{A}\left(i \omega_{n}\right)=\frac{g}{8}\left(\frac{D^{2}}{z_{1}}-z_{0} \ln \left(1-\frac{D^{2}}{z_{0} z_{1}}\right)\right), \\
& \Sigma_{H}\left(i \omega_{n}\right)=-g \frac{z_{1}}{4} \ln \left(1-\frac{D^{2}}{z_{0} z_{1}}\right),
\end{aligned}
$$

where $g=U^{2} A_{c} / \pi v_{F}^{2}=4 U^{2} / D^{2}$ is the dimensionless disorder strength, and $z_{0,1}=i \omega_{n}-\Sigma_{H, A}\left(i \omega_{n}\right)$. Long-range disorder corresponds to $U_{A}(\mathbf{r})=U_{H}(\mathbf{r})=U_{B}(\mathbf{r})$ in Eq. (4), which interestingly yields the same self-energy. Similarly, randomly distributed substitutional impurities with strength $U_{i}$ and concentration $n_{i}$ also give the same self-energy in the Born approximation with $g \sim n_{i} U_{i}^{2}$, though with different numerical prefactors.

In general, these equations can only be solved numerically by, e.g., iteration, but analytical treatment is possible in certain limiting cases. At the Dirac point, after analytical continuation to real frequencies, we obtain

$$
\Sigma_{A}(0)=-i \frac{\sqrt{g} D}{2^{3 / 2}}, \quad \Sigma_{H}(0) \simeq-i \frac{g^{3 / 2} D}{8 \sqrt{2}} \ln \left(\frac{32}{g^{2}}\right) .
$$

The self-consistency equation of $\Sigma_{H}(0)$ in the Born limit parallels closely the self-energy of graphene ${ }^{21,22}$ and $d$-wave superconductors $^{23}$ in the unitary limit. For high energies $(D \gg|\omega| \gg \sqrt{g / 2} D)$, we get for real frequencies,

$$
\begin{aligned}
& \Sigma_{1}(\omega)=g\left(\frac{D^{2}}{8 \omega}-\frac{\omega}{4} \ln \left(\frac{D}{|\omega|}\right)\right)-i \pi \frac{g|\omega|}{8}, \\
& \Sigma_{0}(\omega)=-g \frac{\omega}{2} \ln \left(\frac{D}{|\omega|}\right)-i \pi \frac{g|\omega|}{4},
\end{aligned}
$$

and the real parts are practically negligible with respect to $\omega$.

The knowledge of the self-energies gives immediate access to the sublattice resolved DOS, which reads after analytical continuation as

$$
\rho_{A / H}(\omega)=-\frac{\operatorname{Im}\left[\Sigma_{A / H}\left(i \omega_{n} \rightarrow \omega+i \delta\right)\right]}{\pi U^{2}}
$$

per spin and valley, respectively, $\delta \rightarrow 0^{+}$. The DOS on sublattice $B$ is identical to $A$. The total DOS is then $2 \rho_{A}(\omega)+\rho_{H}(\omega)$, which is dominated by the first term at low energies, the second one only contributes to the linear in energy region for $D \gg|\omega| \gg \sqrt{g / 2} D$.

This reproduces the linear in energy DOS at high energies and weak disorder. Since the flat band exists on sublattice $A$ and $B^{13}$ (i.e., its spinor component on $H$ is zero), the local DOS on sublattice $H$ does not probe its presence in the spectrum, therefore it does not contain the Dirac $\delta$ peak, only the graphene-like linear in energy part. In the presence of disorder, the zero-energy peak in $A$ and $B$ sublattices broadens 


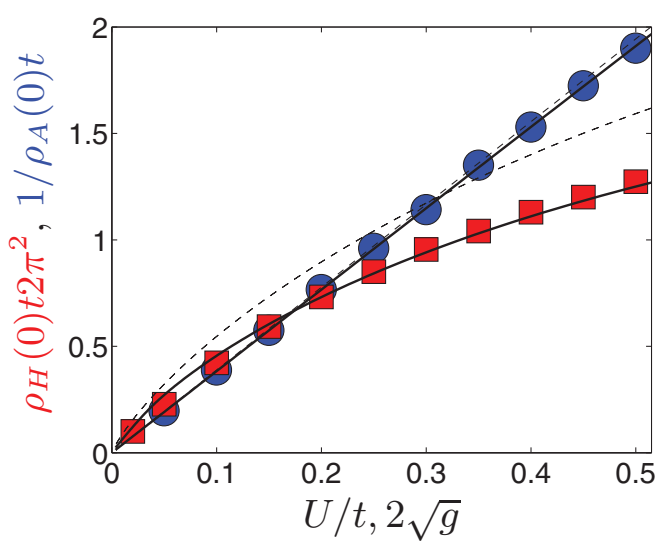

FIG. 2. (Color online) The zero-energy DOS of the $T_{3}$ lattice is shown from KPM, the red squares/blue circles corresponding to sublattice resolved DOS of $H$ and the inverse DOS of $A$, respectively. The solid lines are the results from the SCBA using Eqs. (8) and (11) (with an overall normalization factor as fitting parameter), while the dashed lines stem from the approximate expressions in Eq. (12).

with width $\sqrt{g / 2} D$, while $\rho_{H}(\omega \simeq 0)$ develops a flat plateau. Their heights are

$$
\rho_{A}(0)=\frac{\sqrt{2}}{\pi D \sqrt{g}}, \quad \rho_{H}(0)=\frac{\sqrt{g}}{\pi D 2^{3 / 2}} \ln \left(\frac{32}{g^{2}}\right) .
$$

In spite of the fact that the DOS on sublattice $H$ is graphenelike for the clean system, the effect of disorder on its lowenergy part is completely distinct from that in graphene. ${ }^{21,24}$

The SCBA ignores localization effects, ${ }^{21,22}$ and is expected to be reliable sufficiently far away from the Dirac point, where the clean DOS vanishes. In order to address its validity, we have performed extensive numerical simulation on the dice lattice, containing $2000 \times 2000$ sites with Gaussian distributed potential disorder (with zero mean and $U^{2}$ variance) using the kernel polynomial method (KPM) ${ }^{25}$ The analytical
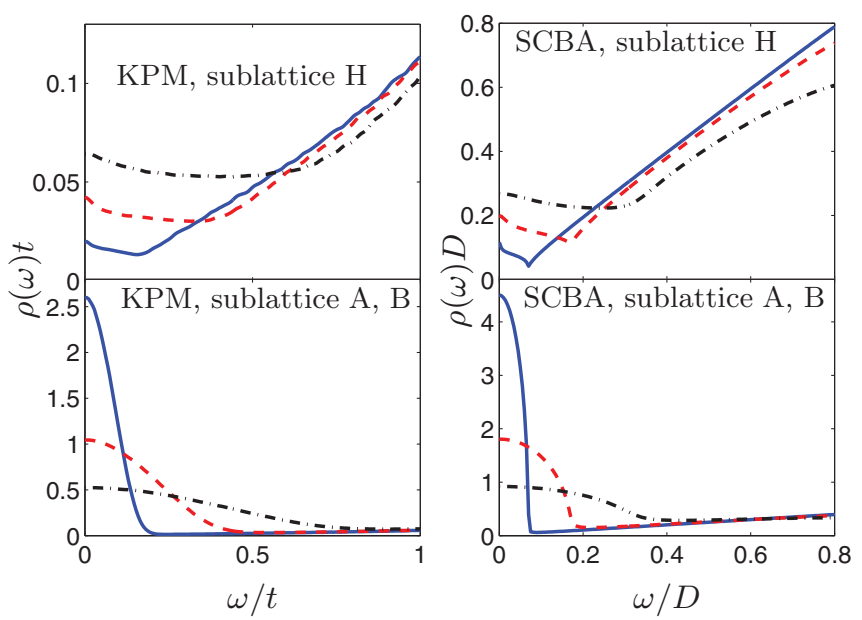

FIG. 3. (Color online) The sublattice resolved DOS is shown from the KPM calculation on the $T_{3}$ lattice for Gaussian disorder with standard deviation $U / t=0.1$ (blue solid), 0.25 (red dashed), and 0.5 (black dash-dotted) in left panel. The right panel visualizes the SCBA results for $\sqrt{g}=0.1$ (blue solid), 0.25 (red dashed), and 0.5 (black dash-dotted). predictions as well as the numerical results are shown in Figs. 2 and 3. The SCBA for Eq. (1) in the continuum limit reproduces all the main features seen in the numerics for the dice lattice, including a dip in the $\mathrm{H}$ sublattice DOS at $\sqrt{g / 2} D$.

\section{DC CONDUCTIVITY}

The dc conductivity is evaluated after dressing the currentcurrent correlation function with impurity lines. The current operator in the $x$ direction is $j_{x}=e v_{F} S_{x}$. Assuming short range scatterers, only self-energy corrections are present, vertex corrections vanish, similarly to the case of graphene..$^{21,24}$ Long-range scatterers would yield finite vertex corrections, though. Then, the dc conductivity is obtained from the Kubo formula

$$
\sigma_{x x}=\lim _{\omega \rightarrow 0} \operatorname{Re} \frac{\chi_{x x}\left(i \omega_{m} \rightarrow \omega+i \delta\right)}{i \omega},
$$

where

$$
\chi_{x x}\left(i \omega_{m}\right)=-\frac{1}{A} \sum_{\mathbf{p}, n} \operatorname{Tr}\left[G\left(\mathbf{p}, i \omega_{n}\right) j_{x} G\left(\mathbf{p}, i \omega_{n-m}\right) j_{x}\right] .
$$

Here, $\omega_{m}$ and $\omega_{n}$ are the bosonic and fermionic Matsubara frequencies, respectively and disorder averaging has already been performed. After some algebraic manipulation, we obtain the dc conductivity at a given chemical potential $\mu$ and at zero temperature as

$$
\sigma_{x x}=\frac{1}{\pi A} \sum_{\mathbf{p}} \operatorname{Tr}\left[G(\mathbf{p}, \mu+i \delta) j_{x} G(\mathbf{p}, \mu-i \delta) j_{x}\right]
$$

per spin and valley, $A=N A_{c}$ is the total area of the system and $\sigma_{y y}=\sigma_{x x}$ and the off-diagonal elements are zero. Performing the momentum integral, and using Eq. (8), we finally get, upon restoring original units,

$$
\sigma_{x x}=2 \sigma_{0} \operatorname{Re}\left[\frac{2 i\left|x_{1}^{2}\right| \operatorname{Re}\left(x_{0}\right)+x_{0} \operatorname{Im}\left(x_{1}^{2}\right)}{\left|x_{1}^{2}\right| \operatorname{Im}\left(x_{0} x_{1}\right) g} \Sigma_{H}(\mu)\right],
$$

where $x_{0,1}=\mu-\Sigma_{H, A}(\mu)$, and $\sigma_{0}=e^{2} / \pi h$ is the universal minimal conductivity per spin and valley of pseudospin- $1 / 2$ Dirac fermions ${ }^{26}$ at half-filling, also found by numerical studies. ${ }^{27}$

For finite doping, after expanding the self energy to second order in $g$ using Eq. (8), and plugging the resulting expressions into Eq. (16), we obtain the conductivity. It becomes practically constant with a weak logarithmic doping dependence for $\sqrt{g / 2} \ll|\mu| / D \ll 1$ as

$$
\sigma_{x x}=\sigma_{0} \frac{8}{3 g}\left(1-\frac{g}{4} \ln \left(\frac{D}{|\mu|}\right)\right),
$$

which agrees qualitatively with that of graphene. ${ }^{21}$ Due to the finite doping, this is dominated by intraband contributions, and the presence of the flat band does not play a role. The weak chemical potential dependence of the conductivity is plotted in Fig. 4, together with the numerical computation of the Kubo formula using KPM. ${ }^{28}$

At the charge neutrality point, Eq. (16) simplifies to

$$
\frac{\sigma_{x x}}{\sigma_{0}}=\ln \left(1-\frac{2 D^{2}}{\Sigma_{H}(0) \Sigma_{A}(0)}\right)=\frac{4 \Sigma_{H}(0)}{\Sigma_{A}(0) g} \simeq \ln \left(\frac{32}{g^{2}}\right),
$$



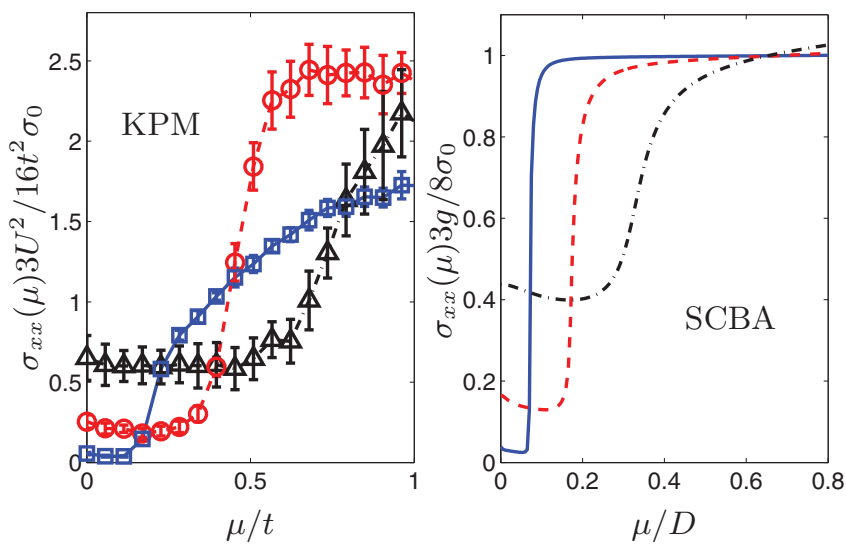

FIG. 4. (Color online) The scaled dc conductivity per spin and valley for $U / t=0.1$ (blue solid), 0.25 (red dashed), and 0.5 (black dash-dotted) is shown as a function of the chemical potential from the KPM calculation of the $T_{3}$ lattice with $2000 \times 2000$ sites (left panel). The right panel visualizes the same quantity calculated from SCBA in the continuum limit for $\sqrt{g}=0.10 .25$ and 0.5 with the same color coding.

and remains roughly unchanged for finite doping in the $|\mu|<\sqrt{g / 2} D$ range, as seen in Fig. 4. The agreement between KPM and SCBA becomes better with increasing system sizes: the average level spacing in the propagating bands is $\sim t / N$, while the broadening of the flat band is $\sim U$. As long as the former is smaller than the latter, the numerics reproduces Eq. (18). Therefore the dc conductivity diverges with decreasing disorder strength as $\ln (1 / g)$ due to the presence of interband transitions between adjacent bands: when bands touch (flat and propagating, see Fig. 1) at the Dirac point, interband transitions are possible in the dc limit.

This can be understood in the constant relaxation time approximation, when an interband transition contributes to $\sigma_{x x}$ at the Dirac point with $\int d^{2} k\left|M_{n, n+1}\right|^{2} \rho_{n}(k) \rho_{n+1}(k)$, where $M_{n, n+1}$ is the matrix element between band $n$ and $n+1$. Here, $\rho_{n}(k)=\Gamma /\left(\Gamma^{2}+\varepsilon_{n}^{2}(k)\right) \pi$ and $\varepsilon_{n}(k)=v_{n}|k|$ are the spectral function and energy dispersion of the $n$th band, respectively, and $\Gamma$ is the scattering rate. In the case of two linearly dispersing bands, this yields $\sigma_{x x} \sim \ln \left(\left|v_{n} / v_{n+1}\right|\right)$. In the case of a flat band, $v_{n}=0$ and the integral is both infrared and ultraviolet divergent for $\Gamma=0$ due to its dispersionless spectrum. While the latter is cured by a natural high energy cutoff $D$, the former requires another energy scale, i.e., the scattering rate, yielding the above logarithmic divergence in Eq. (18), as seen in Fig. 5, confirmed also numerically from the Kubo formula using KPM. Such transitions are, in principle, also present for graphene at the Dirac point but vanish due to the identical velocities in the upper and lower Dirac cones. Note that in spite of the distinct dc conductivities of the pseudospin-1/2 and 1 Dirac-Weyl fermions around zero doping, the zero-frequency limit of their optical conductivites are finite and comparable. ${ }^{13}$

For higher pseudospin generalizations of the Dirac-Weyl equation $^{11,13}$ in Eq. (1), where $\mathbf{S}=\left(S_{x}, S_{y}\right)$ is the matrix representation of an arbitrary spin $S$ (integer or half-integer), interband transitions between two adjacent, propagating bands give a finite contribution the dc conductivity at the Dirac point. For a perfect pseudospin- $S$ Dirac equation with half-integer $S$,

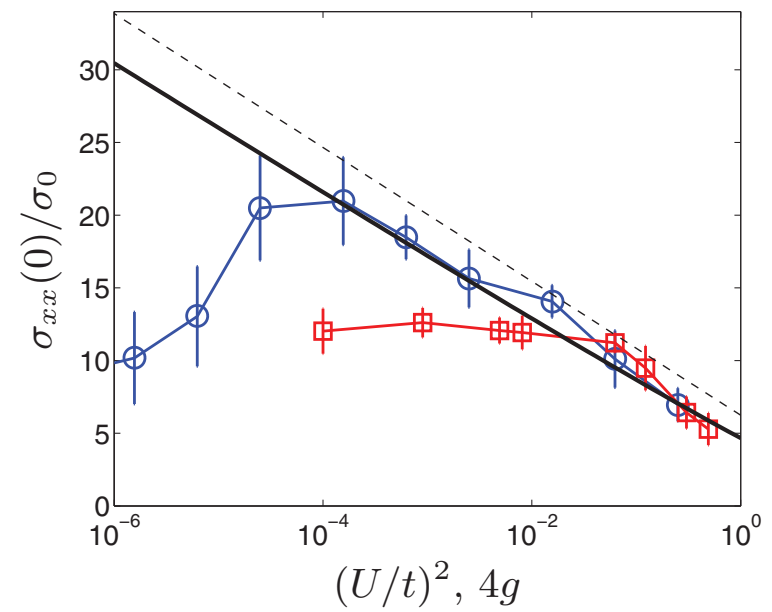

FIG. 5. (Color online) The dc conductivity per cone is shown as a function of the disorder variance from the KPM on the dice lattice for $2000 \times 2000$ (blue circles) and $600 \times 600$ (red squares) sizes, error bars indicated, solid lines connecting the symbols are guides to the eye. The SCBA yields the solid black line from the first equality in Eq. (18), while the thin black dashed line is the approximate $\ln \left(32 / g^{2}\right)$ expression.

the spectrum is $E_{n}(\mathbf{p})=n v_{F}|\mathbf{p}|$ with $n=-S, \ldots, S$, thus all bands are propagating. The electric current operator is $j_{x}=$ $e v_{F} S_{x}$ in the $x$ direction. The universal minimal conductivity is obtained, using Eq. (15) as

$$
\begin{aligned}
\sigma_{S}(\mu=0)= & \frac{\sigma_{0}}{2}\left[\left(S+\frac{1}{2}\right)\left(S+\frac{3}{2}\right)\right. \\
& \left.+\sum_{n=1 / 2}^{S} \frac{S(S+1)-n(n+1)}{2 n+1} \ln \left(\frac{n+1}{n}\right)\right],
\end{aligned}
$$

where the first term stems from intraband processes and the logarithmic terms come from interband transitions between adjacent bands with velocities $n v_{F}$ and $(n+1) v_{F}$. As an example, this gives $\sigma_{3 / 2}=\sigma_{0}\left[3+\frac{3}{4} \ln (3)\right]$ for $S=3 / 2$ and grows with $S^{2}$ for $S \rightarrow \infty$. For integer $S$, the conductivity diverges due to the flat band as $\sim S(S+1) \ln (1 / g)$, similarly to the $S=1$ case in Eq. (18).

\section{SUMMARY}

In conclusion, we have studied the disordered $S=1$ DiracWeyl equation analytically using the SCBA and numerically by the KPM on the dice lattice. The contribution of the flat band is only present in the sublattice resolved DOS for the rim sites. The dc conductivity diverges logarithmically with decreasing disorder due to interband transitions at the band touching point between the flat and propagating bands from the SCBA, showing excellent agreement with KPM. The divergence of the $\mathrm{dc}$ conductivity is a general feature for integer pseudospin-S Dirac-Weyl fermions, and is expected to hold true whenever a propagating and a flat bands touch, i.e., also for the kagome lattice. Their half-integer pseudospin-S counterpart with no flat band possesses a universal minimal conductivity at half-filling, though with a value different from that in graphene. 


\section{ACKNOWLEDGMENTS}

Supported by the Hungarian Scientific Research Fund Nos. K101244, K105149, 75529, and 81492, 108676 , CNK80991, the ERC Advanced Grant, contract 290846, and
ERC Grant No. ERC-259374-Sylo and by the Marie Curie ITN project NanoCTM (FP7-PEOPLE-ITN-2008-234970) and by the Bolyai Program of the Hungarian Academy of Sciences. *dora@eik.bme.hu

${ }^{1}$ K. Sun, Z. Gu, H. Katsura, and S. Das Sarma, Phys. Rev. Lett. 106, 236803 (2011).

${ }^{2}$ T. Neupert, L. Santos, C. Chamon, and C. Mudry, Phys. Rev. Lett. 106, 236804 (2011).

${ }^{3}$ E. Tang, J.-W. Mei, and X.-G. Wen, Phys. Rev. Lett. 106, 236802 (2011).

${ }^{4}$ T. T. Heikkila, N. B. Kopnin, and G. E. Volovik, JETP Lett. 94, 233 (2011).

${ }^{5}$ M. Z. Hasan and C. L. Kane, Rev. Mod. Phys. 82, 3045 (2010).

${ }^{6}$ D. Bercioux, D. F. Urban, H. Grabert, and W. Hausler, Phys. Rev. A 80, 063603 (2009).

${ }^{7}$ R. Shen, L. B. Shao, B. Wang, and D. Y. Xing, Phys. Rev. B 81, 041410(R) (2010).

${ }^{8}$ D. Green, L. Santos, and C. Chamon, Phys. Rev. B 82, 075104 (2010).

${ }^{9}$ V. Apaja, M. Hyrkäs, and M. Manninen, Phys. Rev. A 82, 041402(R) (2010).

${ }^{10}$ D. F. Urban, D. Bercioux, M. Wimmer, and W. Häusler, Phys. Rev. B 84, 115136 (2011).

${ }^{11}$ Z. Lan, N. Goldman, A. Bermudez, W. Lu, and P. Öhberg, Phys. Rev. B 84, 165115 (2011).

${ }^{12}$ H. Watanabe, Y. Hatsugai, and H. Aoki, J. Phys.: Conf. Ser. 334, 012044 (2011).

${ }^{13}$ B. Dóra, J. Kailasvuori, and R. Moessner, Phys. Rev. B 84, 195422 (2011).
${ }^{14}$ N. M. R. Peres, Rev. Mod. Phys. 82, 2673 (2010).

${ }^{15}$ F. Wang and Y. Ran, Phys. Rev. B 84, 241103 (2011).

${ }^{16}$ I. Bloch, J. Dalibard, and W. Zwerger, Rev. Mod. Phys. 80, 885 (2008).

${ }^{17}$ C. D. Giacomo Roati, L. Fallani, M. Fattori, C. Fort, M. Zaccanti, G. Modugno, M. Modugno, and M. Inguscio, Nature (London) 453 , 895 (2008).

${ }^{18}$ W. S. Bakr, J. I. Gillen, A. Peng, S. Fölling, and M. Greiner, Nature (London) 462, 74 (2009)

${ }^{19}$ J.-P. Brantut, J. Meineke, D. Stadler, S. Krinner, and T. Esslinger, Science 337, 1069 (2012)

${ }^{20}$ A. H. Castro Neto, F. Guinea, N. M. R. Peres, K. S. Novoselov, and A. K. Geim, Rev. Mod. Phys. 81, 109 (2009).

${ }^{21}$ P. M. Ostrovsky, I. V. Gornyi, and A. D. Mirlin, Phys. Rev. B 74, 235443 (2006)

${ }^{22}$ I. L. Aleiner and K. B. Efetov, Phys. Rev. Lett. 97, 236801 (2006).

${ }^{23}$ Y. Sun and K. Maki, Phys. Rev. B 51, 6059 (1995).

${ }^{24}$ N. H. Shon and T. Ando, J. Phys. Soc. Jpn. 67, 2421 (1998).

${ }^{25}$ A. Weiße, G. Wellein, A. Alvermann, and H. Fehske, Rev. Mod. Phys. 78, 275 (2006).

${ }^{26}$ J. Cserti, Phys. Rev. B 75, 033405 (2007).

${ }^{27}$ K. Nomura and A. H. MacDonald, Phys. Rev. Lett. 96, 256602 (2006).

${ }^{28}$ A. Ferreira, J. Viana-Gomes, J. Nilsson, E. R. Mucciolo, N. M. R. Peres, and A. H. Castro Neto, Phys. Rev. B 83, 165402 (2011). 